determinasse il residuo e a questo proposito van ricordate l'esperienze del Juraoka, secondo le quali una soluzione di una sostanza in un'ali.'a non dia, almeno in certi casi, alcun residuo, rale a dire non sembra avere quel grado o quelle specie di eterogeneita che secondo il Maxwell determinano il fenomeno. Le cause di questo fenomeno non sono ancora ben chiare, quantunque v' abbia portato molta luce la teoria, che ne diede il Maxwell e che fu verificata in piu casi.

SULL' INTERRUTTORE DI WRHNELT,

del Dott. O. M. CORBINO').

1. Sembra oramai fuori dubbio che nell' interruttore Wehnelt più che l'aziono elettrolitica intervenga l'azione termica della corrente. L' idea, messa già innanzi dallo stesso Wohnelt è stata sviluppata analiticamente in un pregevole studio del Simon ${ }^{2}$ ). Sul numero delle inter ruzioni ha influenza notevole l'autoinduzione amparente del circuito primario.

Tale influenza non può essere determinata (essendo impossibile misurare a priori il valore dell' autoinduzione del primario) che sostituendo all' autoinduzione ignota un' autoinduzione che possa rariare in modo misurabile. Si puó così anche determinare l'influenza che sull' autoinduzione ha la presenza del ferro.

2. I' uso dell' interruttore si presta alle seruenti esperienze:

Esperienza $t^{\mathrm{a}}$. - In un circuito del quale facevano parte una batteria di accumulatori e l' intersutore di Wehnelt, era inserito un l'occhetto di tre strati (filo di $2 \mathrm{~mm}$.) nella cui caviti veniva secondo i casi introdotio un fasccio di fili di ferro dolce avente il diametro di $5 \mathrm{~cm}$. circa. Introducendo il ferro nella caviti, e facendo crescere la resistenza del circuito, il

1) Ini Rend. della R. Acc. dei lincei, Yol. $8,2^{\circ}$ sem., ser. 5., fasc. 12.

2) Wiel. Ann. 68, pag. 27i, 1899. 
numero delle interruzioni docresce, e in tal modo si puó perfino arere una interpuzione ogni cinque o sei minuti secondi. Ciascuna interruzione \& accompagnata da un colpo secco, analogo a quello che si ha con gri ordinari interruttori, mentre l'ago dell' amperometro segna una lieve perturbazione e rifrende subito la posizjono di prima. Aumentando ancora per poco la resistenza, la corrente passa in modo continuo nella raschetta con elettrolisi silenziosa. Cio avriene quando il calore srolto all' anodo non è più sufficiente a provocare l'evaporazione del liquido, perche assorbito dalla intera massa. Facendo crescere, anche di pochissimo, l'intensita limite tra il passdggio continno e il passaggio intermittente della corrente, intensita che chiameremo critica, lo interruzioni aumentano rapidamente di numero. Questi fatti serrono a precisare le idee del Simon, risultando da essi che già un piccolo eccesso sul calore propagato a tutia la massa con l'intensita critica, $\dot{e}$ sufficiente a produre l'interruzione.

Esperienza 2a. - Ripetendo l'osperienza prima senza il mucleo di ferro si puó anche ottenere, per un valore opportuno della resistenza, elettrolisi silenziosa senza interpuzioni; aumentando un poco l'intensita corrispondente, le interpuzioni cominciano meno rumorose che nel caso precedente, per la dimiruita auto-induzione del circuito, na tuttaria abbastanza nette; pero, se mentre passa la corpente senza interruzioni si introduce nella cavita il nucleo di ferro, ancho lentissimamente, cominciano le interruzioni che arrivano a cinque o sei ler secondo quando il ferro è totalmente introdotto. Portando via il ferro, le interruzioni cessano di prodursi. Or che il ferro modilichi la frequenza e il carattere delle interruzioni che si producono anche senza di esso si capisce; ma non mi sembra facile spiegire con la teoria termica, che esso provochi la produzione delle interruzioni nella corrente continua, che sarà solo indebolita durante l'introduzione del ferro, quando la quantita di calore svolta all' anodo non è maggiore di quella di prima.

Esperienza $3^{2}$. - Sullo stesso nucleo di ferro sono avvolti due strati di filo grosso, i quali fan parte di un circuito 
che contiene anche una batteria di 50 accumulatori, l'interr'ltrore di Wehnelt, e una resistenza non induttiva. - I.e interruzioni producono un certo suono di cui si nota l'altezza.

Si sostituisce allora alla rosistenza addizionale una resistenza eguale, avonte peró una induttanza di circa $46 \mathrm{milli-}$ henry (un rocchetto senza ferro). - Il suono si abbassa di una terza minore.

Quindi si dispone attorno al primo nucleo una spira circolare di filo di rame (di $\mathrm{mm} .2,5)$ avente il diametro di $8 \mathrm{~cm}$. Il suono riprende l'altezza di prima; basta cioe la presenza di una spira secondaria per dimiauire la autoinduzione apparente del circuito avolgente il nucleo di una quantita eguale a. 46 millihenry, mentre essa ela di circa 118 millihenry.

Esperienza 4". - Il polo positivo di una batteria di 48 accumulatori è rilegato al fílino di platino dell' interruttore $W^{r}$;

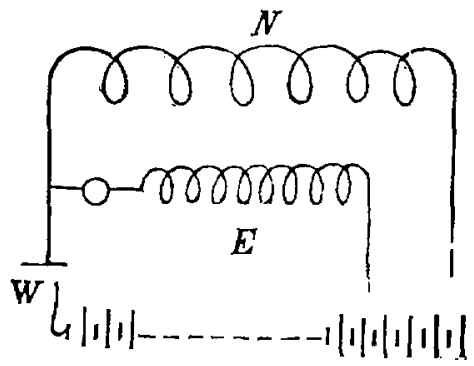

all' allro elettrodo di questo il conduttore si biforca: in una derjazione è inserita la solita bobina $N$ che avolge il nucleo di fili di ferro; nell' altra un anperometro ed una elettrocalamita Ruhmkorff F. L'altro estremo del circuito E è rilegato con la placca negativa del $6^{\circ}$ accumulatore, mentre quello del cir" cuito $N$ puó rilegarsi a volontá, o alla stessa placca, o alla negativa del $1^{0}$ accumulatore. Tel primo caso la coprente interrotta dall' apparecchio di Wehnelt si biforca nei due circuiti a nella branca $\mathrm{E}$ si ha una intensita modia di 6,5 anpère. Nel secondo caso si sovrappongono nella branca E la corrento variabile di prima e la corrente continua dovuta ai cinque accumulatori interposti tra i due circuiti derivati.

Questit seconda corrente, oppostiz alla prima, aveva per sé sola una intensita costante di $6, \dot{z}$ ampère. Per la sovrapposi- 
zione delle due si hanno nella branca $\mathrm{E}$ delle correnti alternate con circolazione noi due sensi di quantità eguali di elettricità ; l'ago dell' amperometro resta a zero, e la elettrocalamita, che si magnetizzava fortemente per il prassaggio dell'una o dell'altra delle due correnti, resta completamente smagnetizzata, come risulta sia dalla mancanza di attrazione su un fascetto di ferro dolce, sia dalla assenza del fenomeno di Faraday (rotazione magnetica del piano di polarizzazione della luce).

Disponendo i contatti in guisa che le correnti opposte, di intensità medie eguali, si sovrappongano non più nella branca $\mathrm{E}$ ma nella branca $\mathbb{N}$, nella quale si trova, come si disse, un nucleo di fili di ferro, l' attrazione sul ferro dolce viene di nolto indebolita, ma non annullata. Siccome in quest' ultimo caso erano evitato le correnti indotte nella massa, si potrebbe a prima vista attribuire la diminuzione di magnetizzazione avuta con la sovrapposizione delle due correnti a un ritardo della magnetizzazione del nuicleo, per cui questo non seguirebbe le variazioni rapidissime del campo magnetizzante.

Di tale ritardo, presumibile dopo i risultati del Maurian ${ }^{1}$ ) e di altri, non è necessario invocare l' esistenza per spiegare il fatto osservato, poichè la steșsa cosa si sarebbe trovata anche se il ferro seguisse senza ritardo le vicissitudini del campo magnetizzante. Ed infatti la coside tta for za portante del nucleo essendo proporzionale al quadrato dell intensita di magnetizzazione, sarà anche proporzionale al quadrato della intensità della corrente, se si suppone costante la suscettività del ferro. Con una corrente periodicamente variabile l' attrazione si potrà poi ritenere proporzionale all' espressione:

$$
\frac{1}{\mathrm{~T}}\left[\int_{0}^{\mathrm{T}} i^{2} d t-\mathrm{I}^{2} \mathrm{I}^{\top}\right]
$$

ove 'T è il periodo di variazione della corrente; cioè sarà eguale alla differenza tra l'attrazione che sarebbe prodotta

1) A nuales do Ch. o Phys. (7), 14, pag. 282, 1898. 
dalla corrente rariabile e quella che sarebbe prodotta dalla corrente costante.

Si spiega così la diminuzione dell' attrazione nella massima parte del periodo.

Poichè anche all' effetto Joule si possono applicare (anzi rigorosamente) queste considerazioni, se ne deduce che con una disposizione analoga alla precedente si potrebbe in un circuito (ad es. il primario di un rocchetio) avere le stesse variazioni nellar intensità della corrente con riscaldamento minore che nella disposizione ordinaria.

Esperienaa $5^{n}$. - Inviando nel primario di un trastormatore la corrente interiotta con l'apparechio di Wehnelt, si ha nel secondario rilegato all elettrocalamita una corrente allernatil di circa 3 ampère d'intensità eflicace, senza magnetizzazione sensibile del nucleo dell' elettrocalamita, mentre una corrente continù di pochi decimi di ampère produce una magnetizazione notevole.

Questo fatto è da attribuirsi interamente alle correnti inIotte negli strati superficiali del nucleo massiccio. Infatti, se i) secondario del trasformatore è rilegato al primario di un rocchetto di Ruhmkorff di media grandezza (nel quale il nucleo di forro è frazionato in fili) quando il secondario di questo è chiuso metallicamente, il nucleo non si magnetizza fortemente perché, come è noto, i tlussi magnetici creati dal primario e dal secondario sono* opposti e di intensità quasi eguale; se però si portano gri estremi del secondario a grande distanza in modo che tra loro non si abbiano scintille, il nucleo manifesta nettamente una non debole magnetizazione.

Esperienza $6^{21}$. - Un rocchettino è inserito nel circuito del secondario del trasformatore chiuso su una resistenza qualunque.

l.a forma delle curve rappresentanti la intensita della corrente in funzione del tempo si è studiata con un tubo di Braun diretto perpendicolar'mente all' asse del l'occhettino, e nel quale passa la scarica di una macchina roepler a quaranta dischi.

Il cerchietto fluorescente prodotto dalla scarica viene esa. minato con uno specchio girante; le curve vedute nello specchio hanno all' incirca la forma disegnata qui sotto. 
Ia teoria permette, facendo qualche ipotesi che si presenta come abbastanza verosimile, di trovare una formula che dà la

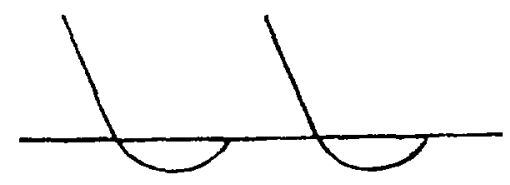

intensità nel secondario in funzione del tempo o delle costanti relative ai circuiti.

Siano $\mathrm{I}, \mathrm{R}$ la induttanza e la resistenza del primario, $E$ la for'za elettromotrice agente in esso; $L^{\prime}, R^{\prime}$ la induttanza e la resistenza del secondario nel quale si suppone non esistano altre forze elettromotrici ; $\mathrm{M}$ il coefliciente di induzione mutua dei due circuiti, $i$ ed $i^{\prime}$ le intensità delle rispettive correnti.

Le leggi dell' induzione dànno

(1)

$$
\left\{\begin{array}{l}
\mathrm{L} \frac{d i}{d t}+\mathrm{M} \frac{d i^{\prime}}{d t}+\mathrm{R} i-\mathbf{E}=0 \\
\mathrm{I}^{\prime} \frac{d i^{\prime}}{d t}+\mathrm{M} \frac{d i}{d t}+\mathrm{R}^{\prime} i^{\prime}=0
\end{array}\right.
$$

Queste equazioni ammettono, come è noto, degli integrali generali dati dalle relazioni

$$
\left\{\begin{array}{c}
\mathrm{R} i-\mathrm{E}=\mathrm{A} e^{p t}+\mathrm{B} e p^{\prime} t \\
\mathrm{R}^{\prime} i^{\prime}=\mathrm{A}^{\prime} e e^{p t}+\mathrm{B}^{\prime} e \rho^{p^{\prime} t}
\end{array}\right.
$$

Ore $\mathrm{A}, \mathrm{B} ; \mathrm{A}^{\prime}, \mathrm{B}^{\prime}$, sono costanti determinabili con I' assegnare valori particolari al tempo, e $\rho, \rho^{\prime}$ sono le due radici, entrambe negative, dell' equazione

$$
\left(L L^{\prime}-M^{2}\right) \rho^{2}+\left(I^{\prime} R+L R^{\prime}\right) \rho+R R^{\prime}=0 .
$$

Le costanti jossono determinarsi dietro considerazioni speciali.

Si deduce dalle (1) e (2) per la corrente secondaria

$$
A^{\prime}\left(p^{\prime}-p\right)=\frac{M}{L^{\prime}}\left[R R^{\prime}+\left(L R^{\prime}+L^{\prime} R\right) p\right] I
$$


e

$$
i^{\prime}=\frac{\mathrm{A}^{\prime}}{\mathrm{R}^{\prime}}\left(e \mathrm{p}^{t}-e^{p^{\prime} t}\right)+\frac{\mathrm{M}}{\mathrm{L}^{\prime}} \mathrm{I} e p^{\prime} t
$$

dove I indica il valore dell' intensitá nel primario all' istanto della rottura.

Il valore di I è anch' esso ignoto. Il Simon ammette che nel primario la corrente raggiunga, anzi presto, il valore che corrisponde alla forza elettromotrice $\mathrm{F}$ e alla resistenza $\mathrm{R}$, e ciò è probabile quando $R$ è piuttosto grande, e la presenza del secondario attenua l' autoinduzione apparente del primario.

In tali condizioni, ciò̀ ammettendo che sia

$$
I=\frac{E}{R}
$$

le (3) $\theta$ (4) divengono

$$
\begin{gathered}
\mathrm{A}^{\prime}\left(\rho^{\prime}-\rho\right)=\stackrel{\mathrm{ME}}{\mathrm{L}^{\prime} \mathrm{R}^{\overline{2}}}\left[\mathrm{RR}^{\prime}+\left(\mathrm{I} \mathrm{R}^{\prime}+\mathrm{L}^{\prime} \mathrm{R}\right) \rho\right] \\
i^{\prime}=\frac{\mathrm{A}^{\prime}}{\mathrm{R}^{\prime}}\left(e \rho^{\prime}-e^{\rho^{\prime} t}\right)+\frac{\mathrm{ME}}{\mathrm{L}^{\prime} \mathrm{R}} e \rho^{\prime} t
\end{gathered}
$$

Queste formole risolvono il problema. Esse valgono per ciascun periodo successiro.

\section{IIBRI NUOVI}

\section{LETTURS SCIENTIFICHE POPOLARI,}

$d i$ E. MACH.

(Torino, Fratelli Bosca, editori, 1900).

E un eleganto volume della «Piccola biblioteca di scienze moderne » pubblicata per cura degli editori Bocca di Torino.

In questo libro tradotto dal tedesco da A. Bongiovanni, sono trattati in modo molto semplice e veramente popolare diversi importanti argomenti. Esso è diviso in tredici capitoli in ciascuno dei quali è trattato un tema diverso. 